Original Article

\title{
COMMUNITY PHARMACIST-LED NEW MEDICINE SERVICE FOR PATIENTS WITH A LONG TERM MEDICAL CONDITION: A CROSS-SECTIONAL STUDY
}

\author{
EJAZ CHEEMA ${ }^{1,2}$, PAUL SUTCLIFFE ${ }^{1}$, DONALD RJ SINGER ${ }^{3}$ \\ ${ }^{1}$ Warwick Medical School, Gibbet Hill Campus, University of Warwick, Coventry, United Kingdom, ${ }^{2}$ College of Pharmacy, Abdeyah Campus, \\ Umm-Al-Qura University, Makkah, Saudi Arabia, ${ }^{3}$ Fellowship of Postgraduate Medicine, 12 Chandos Street, London, United Kingdom \\ Email: e.cheema.1@warwick.ac.uk \\ Received: 03 Feb 2016 Revised and Accepted: 29 Apr 2017
}

\begin{abstract}
Objective: This study assessed the impact of the new medicine service (NMS) on medication use in patients starting a new medication for a longterm medical condition in the United Kingdom (UK).

Methods: A cross-sectional study was conducted in community pharmacies in the West Midlands area for three months from July to September 2012. The drug therapies/agents included in the study were antihypertensive, antidiabetics, anti-asthmatics and antiplatelet/anticoagulants.

Results: 20 community pharmacists completed questionnaires related to 285 patients (160 female and 125 male). 0 n the first NMS assessment, 82 patients reported drug-related problems including adverse effects and incorrect use of medications. Of these 82 patients, 58 received pharmacists' advice and 24 did not receive any advice. At the NMS follow up 39 (67\%) of the 58 patients who received pharmacists' advice reported resolution of their drug-related problems while only four (17\%) of the 24 patients who did not receive pharmacists' advice reported resolution of their problems (odds ratio 10.2, 95\% CI 3.0-34.2 p<0.0001). The improvement in the correct use of medications by patients reported in this study for example by improving the inhaler technique of asthmatic patients is expected to have important implications for improving the healthcare outcome of patients with long-term conditions.
\end{abstract}

Conclusion: This study provides support for the NMS as an opportunity to improve detection of adverse effects and improve the incorrect use of medicines by patients. Further research is needed to address the policy implications of the NMS, including analyses of the clinical and costeffectiveness of this service, and the sustainability of this form of pharmacist intervention in the long-term in clinical practice.

Keywords: New medicine service, Community pharmacist, Adverse drug reactions

(C) 2017 The Authors. Published by Innovare Academic Sciences Pvt Ltd. This is an open access article under the CC BY license (http://creativecommons.org/licenses/by/4.0/) DOI: http://dx.doi.org/10.22159/ijpps.2017v9i6.17464

\section{INTRODUCTION}

Research suggests that approximately $50 \%$ of patients with longterm medical conditions do not take their medications as prescribed [1]. A report published by the American Heart Association in 2014, reported that $24 \%$ of patients who were admitted for a heart attack did not fill their medications within seven days of hospital discharge, and $34 \%$ patients with myocardial infarction discontinued one of their medications within $30 \mathrm{~d}$ of hospital discharge [2]. Poor adherence to medication by patients is not only associated with increased morbidity and death but is also associated with a significant financial impact on the health services through medicines waste [3]. A study evaluating the scale, costs and causes of medicine waste in England reported that the cost of medicine waste is estimated to be around $£ 250-£ 300$ million per year in England [4].

In the UK, community pharmacists are encouraged to play an active role in clinical services aimed at improving patient adherence with their medications. The NMS is based on actions and advice arising from the subjective assessment of patients who have been newly prescribed a medication for a long-term condition, combined with follow-up to address any concerns or issues patients may have once they have started using the new medicine. Issues identified within the NMS may include the ineffective use of medications and detection of adverse drug reactions (ADRs) that may affect compliance to medications.

Proof of concept research $[5,6]$ was used in the development of this new service, showing how interventions by a pharmacist can help to improve patient adherence to their medications. Patients who used a pilot telephone-based pharmacist service experienced fewer medication problems and made less use of the National Health Service (NHS), reducing both costs of healthcare and GP time [6]. The NMS can be provided to patients who have been newly prescribed any of four drug therapies/agents: antihypertensive, antidiabetics, anti-asthmatics and antiplatelet/anticoagulants [7]. The new medication could have been prescribed for a newly diagnosed condition or an existing long-term medical condition.

Eligible patients receive the NMS in two stages: an intervention stage within two weeks of starting the new medication, conducted in the pharmacy or over the telephone, and a follow-up stage three weeks later [7]. A recently conducted evaluation work carried out on behalf of the Department of Health, UK has concluded that the implementation of the NMS has been very successful in the UK and over $90 \%$ of community pharmacies have now offered this service [8]. This study demonstrated that NMS had significantly improved medicine adherence in patients by $10 \%$ [8]. However, this study did not explain the reasons or factors which contributed to the improvement in medication adherence. The aim of this study was to extend the previous assessment of the NMS on medication adherence by defining the reports of concerns about medication safety, efficacy and use, and the resolution both of adverse effects of drugs and incorrect use of medicines by patients.

\section{MATERIALS AND METHODS}

Evaluation of the NMS was conducted by administering a questionnaire to the community pharmacists based in the West Midland area for three months from July to September 2012. A 12item questionnaire was adopted from the validated set of worksheets produced by the pharmaceutical services negotiating committee (PSNC) for pharmacists to apply the NMS in practice [7]. Pharmacists were required to complete the questionnaire with data from one single completed NMS patient for all four drug therapies/agents of NMS where possible. The drug therapies/agents included in the study were antihypertensive, antidiabetics, antiasthmatics and antiplatelet/anticoagulants. 
Section A of the questionnaire required the pharmacists to provide details of their first consultation with the patients (intervention stage) including patient's age and gender, name and dosage of the medicine used by patient, any medication-related problems reported by the patient including adverse effects and incorrect use of medicine and any subsequent action taken or advice provided by pharmacists. Examples of possible actions taken by pharmacists included referral of the patient to the doctor and submission of a yellow card to Medicines and Healthcare products Regulatory Agency (MHRA) in the case of an adverse effect reported by the patient. This section also recorded details of any lifestyle advice provided to patients. Section B required the pharmacists to report details of their second consultation with the patients (follow-up stage). It recorded the outcome of the advice provided by pharmacists at the intervention stage on medication use by patients.

A total of 120 community pharmacists were invited to take part in the study through the Dudley local pharmaceutical committee (LPC) who supported and endorsed this study. Statistical analysis was performed with SPSS (IBM version 22). Summary descriptive statistics were generated from the questionnaire data using SPSS.

\section{Ethical approval}

Based on the criteria of the National Research Ethics Service, UK the project falls within the category of service evaluation; therefore ethics approval was not required. No personal data from patients or pharmacists was collected. All collected data was kept strictly confidential. The procedures for handling, processing, storage and destruction of the data complied with the Data Protection Act 1998.

\section{Interventions}

Interventions considered in this study were those expected to be received by patients within NMS from community pharmacists as per service specifications produced by the PSNC [7]. These interventions included both drug/medicine related and nonmedicine/drug related interventions. The drug-related interventions included an assessment of patient adherence to the new medication, identification of any problems the patient may be having with their new medication(s) and exploration of possible solutions to reported problems. The non-medication/drug related interventions included lifestyle changes such as advice offered by pharmacists on healthy eating, advice on weight management, smoking, alcohol consumption, physical activity and advice on sexual health. These interventions were conducted either by telephone or in pharmacy consultation rooms.

\section{RESULTS}

20 community pharmacists based in 14 pharmacies returned the completed questionnaires. The questionnaires had anonymous data of 295 patients recruited by the participating pharmacists in three months from July 2012 to September 2012. 10 questionnaires were excluded from this study due to missing data. Of the 285 patients included in the study, 160 (56\%) patients were female and 125 (44\%) were male.

\section{Medications}

Antihypertensive were the most common drug therapy/agent among the four drug therapies/agents considered in the study with $145(51 \%)$ patients receiving a new anti-hypertensive medication. They were followed by anti-asthmatics with 88 (31\%) patients receiving a new medication, while antidiabetics and antiplatelet/ anticoagulants accounted for $37(13 \%)$ and 15 (5\%) of patients respectively. For the 285 new medications recorded on these questionnaires, calcium channel blockers (mainly amlodipine) and ace-inhibitors (mainly ramipril) were the two main classes of medications used (table 1).

Table 1: List of medication classes used in NMS interventions

\begin{tabular}{|c|c|c|}
\hline Class of medication & Medical condition & No. of medications \\
\hline Ace inhibitors & Hypertension & 40 \\
\hline Calcium Channel blockers & Hypertension & 45 \\
\hline Angiotensin receptor blockers & Hypertension & 20 \\
\hline Beta blockers & Hypertension & 15 \\
\hline Diuretics & Hypertension & 15 \\
\hline Alpha blockers & Hypertension & 7 \\
\hline Renin inhibitors & Hypertension & 1 \\
\hline centrally acting drugs & Hypertension & 2 \\
\hline Short acting $\beta 2$-adrenergic receptor agonist & Asthma/COPD & 36 \\
\hline Long acting $\beta 2$-adrenergic receptor agonist & Asthma/COPD & 13 \\
\hline Corticosteroid+Long acting $\beta 2$-adrenergic receptor agonist & Asthma/COPD & 19 \\
\hline Corticosteroid & Asthma/COPD & 17 \\
\hline Leukotriene receptor antagonist & Asthma/COPD & 3 \\
\hline Biguanide & Diabetes & 20 \\
\hline Sulfonylurea & Diabetes & 7 \\
\hline Alpha glucosidase inhibitor & Diabetes & 1 \\
\hline GLP-1 receptor agonists & Diabetes & 1 \\
\hline DPP-4 inhibitors & Diabetes & 3 \\
\hline Insulin Analog & Diabetes & 4 \\
\hline Biguanide+DPP-4 inhibitors & Diabetes & 1 \\
\hline Antiplatelet & Antiplatelets/Anticoagulants & 9 \\
\hline Anticoagulant & Antiplatelets/Anticoagulants & 6 \\
\hline
\end{tabular}

\section{Quantitative description of NMS interventions}

A total of 285 NMS consultations were recorded by the participating pharmacists in the three-month study period. 279 of these consultations were conducted over the telephone while the remaining six were conducted at the pharmacy premises (pharmacy consultation rooms). Pharmacists recorded 269 patients as adherent (self-reported by patients) to their medicines, while 16 patients were found to be non-adherent at the intervention stage. The reasons for non-adherence included one patient getting concerned after reading the leaflet, one patient been admitted to hospital and 14 patients been advised by their GP to stop taking their medicine.

\section{Description of ADRs recorded by pharmacists}

$51(18 \%)$ of the 285 patients reported ADRs with their newly prescribed medications. The incidence of ADRs was reported to be highest for anti-diabetic medications and antihypertensive medications (25\% each) while medications used for asthma or COPD and antiplatelet/anticoagulants had the lowest incidence of reported ADRs (7\% each). Common ADRs reported by patients included a dry cough, swollen ankles, headaches, and dizziness. 22 patients who reported ADRs with their new medications were referred to their GP by the pharmacists. There is a specific requirement on the NMS worksheets to record suspected ADR through yellow card reporting 
[7]. No yellow cards were reported as submitted to the medicines and healthcare products regulatory agency (MHRA).

\section{Description of incorrect use of medications by patients}

$27(10 \%)$ of patients included in the study were not using their new medications correctly when assessed by pharmacists at the intervention stage. In 17 of these patients, the incorrect use of medication was related to asthma or COPD. In eight patients, there were concerns about the use of anti-hypertensive medications (seven were missing doses and one was taking medication at the wrong time). One patient was not taking his anti-diabetic medication as prescribed and the remaining patient was not taking her antiplatelet medication as prescribed.

\section{Description of healthy lifestyle advice given by pharmacists}

$65(23 \%)$ patients were reported to receive advice on a healthy lifestyle from pharmacists. The most common lifestyle advice given to patients was related to diet and nutrition (fig. 1).

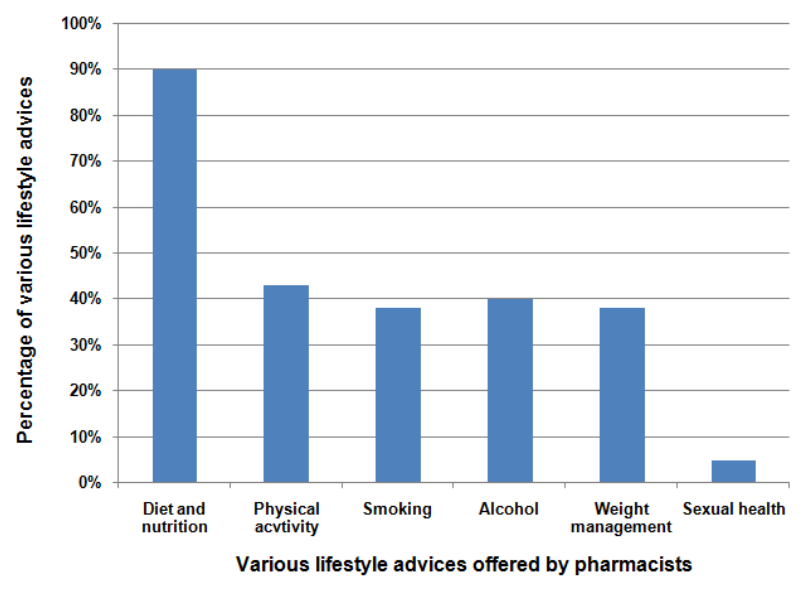

Fig. 1: Proportion of lifestyle advice given to 65 patients by pharmacists

\section{Effectiveness of pharmacists' interventions}

A total of 82 patients reported drug-related problems including adverse effects and incorrect use of their medications at the intervention stage. 58 of the 82 patients were reported to have received advice from pharmacists while 24 patients did not receive advice from the pharmacists. $39(67 \%)$ of the 58 patients who received pharmacist advice reported resolution of their drug-related problems at the followup stage ( 16 due to ADRs and 23 due to the incorrect use of medications) while 19 patients did not report resolution of their problems at the follow-up stage. Of the 24 patients who did not receive advice from pharmacists, only four $(17 \%)$ of patients reported resolution of their problems at follow-up stage (three problems due to ADRs and one related to incorrect use of medicines) while the remaining 20 patients did not report any resolution of their problems (odds ratio $10.2,95 \% \mathrm{CI}$ 3.0-34.2 $\mathrm{p}<0.0001)$.

\section{DISCUSSION}

Long-term medical conditions are imposing an increasing burden on health care systems. In England alone, around 15 million people are estimated to have a long-term condition requiring medication and other therapies [9]. The findings of this study demonstrate the important contributions of community pharmacists within the NMS including both detecting a high rate of ADRs attributed to new medications and incorrect use of medications as common, addressable problems.

The largest category of drug-related issues identified by pharmacists in this study was reported to be the incorrect use of medications by patients. It is noteworthy that pharmacists identified $10 \%$ of the patients with incorrect use of their medications. Further research is needed to investigate two major questions arising from this aspect of service evaluation: how to prevent the initial occurrence of medication-related problems such as the adverse effects and efficacy issues; and, what would make interventions by community pharmacists more effective when aiming to resolving medicationrelated problems?

A high prevalence of ADRs (18\%) was reported by patients during NMS consultations with the pharmacists. The incidence of ADRs was reported to be highest (25\% each) for antidiabetic medications and anti-hypertensive medications. A high incidence of ADRs with antidiabetic $(17 \%)$ and anti-hypertensive medications (15\%) was previously reported in the PSNC NMS summary data report [10]. 22 patients who reported ADRs in this study were referred to their GP by the pharmacists. A weakness of the NMS is that no outcome data is recorded for NMS interventions by a pharmacist. Within the current service specifications of the NMS, there is an opportunity to establish contact with an individual patient by conducting a medicines use review after six months. This is a potential weakness of the NMS as lack of validation means that pharmacists are unaware if their advice to patients and GPs is acted upon. As evident from the findings of this study, some NMS consultations will necessitate recommendations being made to GPs e. g. a change in formulation or inhaler device or perhaps because an adverse drug reaction is reported. In the absence of any feedback on their recommendations, community pharmacists would be unaware of the impact of their clinical advice to prescribers. A separate study would be needed with ethical approval to approach and track patients to obtain objective evidence of the effectiveness of the NMS in practice.

The NMS provides a specific prompt to report ADRs using the national Yellow Card reporting system [7]. However, despite recording 51 ADRs, none of the pharmacists who took part in this study reported submitting a Yellow Card for a suspected ADR. This finding is in contrast to the findings of previous evidence which suggested that following the introduction of the NMS in October 2011, over 700 new Yellow Cards were reported by pharmacists over a 12 mo period [11]. Prompt reporting of suspected ADRs is fundamental in the post-marketing surveillance of medicines and helps in ensuring medicine safety [12]. Pharmacists should, therefore, use the opportunity provided in the NMS to report suspected ADRs to the MHRA. Evidence suggests that increased awareness and education about ADRs can improve the knowledge and perception of healthcare professionals including pharmacists towards ADR reporting [13-15].

Successive recent governments in the UK have initiated schemes for extensions in the role of community pharmacists, through independent prescribing, medication use reviews, and health promotion [16-18]. The NMS continues this strategy, with providing lifestyle advice as a major component [7]; in addition to identifying and managing ADRs, and ensuring that medications are being used appropriately. However, only one in four patients in this analysis were reported to have been given lifestyle advice by pharmacists. Lifestyle advice should be provided at both interventions and followup stage of NMS. Previous systematic reviews and meta-analysis have demonstrated that community pharmacists can significantly improve blood pressure control of hypertensive patients by giving advice related to medications as well as advice on lifestyle [19-23].

Although the NMS consultations can be conducted both over the telephone and in the consultation room, telephonic consultations were the most popular method of NMS contact in this study. There is some evidence to suggest that telephonic consultations can achieve better compliance to treatment as compared to face-to-face consultations [24]. Telephonic consultations can be a preferable method of contact both for patients who do not live close to the pharmacy and for pharmacists as it can allow them to conduct consultations at less busy times [25].

The identification of the incorrect use of medicines by asthmatic patients reported in this study would have important implications for improving the healthcare outcome of patients with asthma. Several studies involving community pharmacy-led asthma interventions have demonstrated a positive impact on patients' asthma-related quality of life and peak expiratory flow rates [26-30]. 
In Finland, a nationwide asthma programme was introduced whereby a community pharmacist helps the prescriber in delivering guidance to asthmatic patients on their medication [28]. Similarly, in Denmark, an asthma-specific pharmaceutical care programme was associated with a reduction in inhalation errors and improved drug prescribing [31]. There is an obvious scope to extend initiatives like NMS to other countries as it has a great potential to improve new medication use in patients with long-term conditions. However, more work involving an active participation from community pharmacists needs to be done to demonstrate the clinical value of this service.

This study has several key limitations. Although this study demonstrated the contribution of NMS in detecting ADRs and incorrect use of medicines by patients; these findings could have been explained by confounding. This study had no control group and therefore, it cannot be assumed that the positive impact on patient healthcare outcomes reported in this study was produced by pharmacists' interventions. Another limitation of this study was the lack of objective clinical data on patient outcomes. However, it should be pointed that like medicines use review (MUR), NMS is a review of medicines use and is not a clinical review. Recording clinical data for patients is outside the scope of NMS and is a weakness of the NMS in its current format.

\section{CONCLUSION}

This study provides support for the NMS as an opportunity to improve detection of adverse effects and improve the incorrect use of medicines by patients. Further research is needed to address the policy implications of the NMS, including analyses of the clinical and cost-effectiveness of this service, and the sustainability of this form of pharmacist intervention in the long-term in clinical practice.

\section{ACKNOWLEDGMENT}

We would like to thank the Dudley LPC for its support in data collection.

\section{AUTHOR CONTRIBUTION}

Author Singer contributed to the study conception and design. Author Cheema contributed to the acquisition of data, analysis and interpretation of data. Author Sutcliffe contributed to the preparation of the manuscript as well as to the critical revision of the manuscript.

\section{CONFLICT OF INTERESTS}

\section{Declared none}

\section{REFERENCES}

1. Vrijens B, Vincze G, Kristanto P, Urquhart J, Burnier M. Adherence to prescribed antihypertensive drug treatments: longitudinal study of electronically compiled dosing histories. Br Med J 2008;336:1114-7.

2. American Heart Association. A Tough Pill to Swallow: Medication Adherence and Cardiovascular Disease; 2014. Available from: www.Heart.org/idc/groups/heart-public/ @wcm/@adv/documents/downloadable/ucm_460769.pdf. [Last accessed on 27 Feb 2017].

3. Viswanathan M, Golin CE, Jones CD, Ashok M, Blalock SJ, Wines $\mathrm{RC}$, et al. Interventions to improve adherence to selfadministered medications for chronic diseases in the United States: a systematic review. Ann Intern Med 2012;157:785-95.

4. Trueman P, Taylor DG, Lowson K, Bligh A, Meszaros A, Wright D, et al. Evaluation of the Scale, Causes and Costs of Waste Medicines; 2010. Available from: http://eprints.pharmacy.ac.uk/ 2605/1/ Evaluation_of_NHS_Medicines_Waste__web_publication_version .pdf. [Last accessed on 19 Sep 2016]

5. Barber N, Parsons J, Clifford S, Darracott R, Horne R. Patients' problems with new medication for chronic conditions. Qual Saf Health Care 2004;13:172-5.

6. Clifford S, Barber N, Elliott R, Hartley E, Horne R. Patientcentred advice is effective in improving adherence to medicines. Pharm World Sci 2006;28:165-70.
7. Pharmaceutical Services Negotiating Committee. Service specification-New Medicine Service (NMS); 2013. Available from: http://psnc.org.uk/wp-content/uploads/2013/06/NMSservice-spec-Aug-2013-changes_FINAL.pdf. [Last accessed on 18 Aug 2016].

8. Elliott RA, Boyd MJ, Waring J, Barber N, Mehta R, Chuter A. A randomised controlled trial and economic evaluation with qualitative appraisal comparing the effectiveness and costeffectiveness of the New Medicines Service in community pharmacies in England. A Department of Health Policy Research Programme Project; 2014. Available from: http://www.nottingham.ac.uk/ pazmjb/nms/downloads/rep ort/index.html. [Last accessed on 19 Sep 2016]

9. Department of Health. Improving the health and well-being of people with long-term conditions. World class services for people with long-term conditions: information tool for commissioners; 2010. Available from: http:// www.yearofcare.co.uk/sites/default/files/pdfs/dh_improving \%20the $\% 20$ h\%26wb\%20of\%20people\%20with\%20LTCs.pdf. [Last accessed on 22 Aug 2016]

10. Pharmaceutical Services Negotiating Committee. Evaluation of evidence provided by pharm outcomes New Medicine Service Data; 2012. Available from: http://psnc.org.uk/wp-content/ uploads/2013/07/PO_NMS_data_evaluation_Nov_2012_full_re port.pdf. [Last accessed on 22 Aug 2016]

11. Jadeja M, McCreedy C. Positive effect of new medicine service on community yellow card reporting. P J 2012;289:159-60.

12. Medicines and Healthcare products Regulatory Agency. Best practice in reporting of individual case safety reports (ICSRs); 2011. Available from: http://www.mhra.gov.uk/home/ groups/comms-ic/documents/publication/con108808.pdf. [Last accessed on 18 Aug 2016]

13. Adepu R, Srikanth MS, Nagaraj S. Impact of an educational intervention on knowledge, attitude, and practices of urban community pharmacists toward adverse drug reaction reporting in a south Indian city. Asian J Pharm Clin Res 2016;9:140-4.

14. Ravinandan AP, Achutha V, Ramani VK, Kumar SL. Study of knowledge, attitude and practice of pharmacist towards adverse drug reaction reporting in davangere city. Asian J Pharm Clin Res 2015;8:262-5.

15. Sivadasan S, Sellappan M. A study on the awareness and attitude towards pharmacovigilance and adverse drug reaction reporting among nursing students in a private university, Malaysia. Int J Chem Pharm Res 2015;7:84-9.

16. Department of Health. Choosing health through pharmacy-a programme for pharmaceutical public health 2005-2015. London: Department of Health; 2005.

17. Coggans N, McKellar S, Bryson S, Parr R. Evaluation of health promotion development in Greater Glasgow Health Board community pharmacies. P J 2001;266:514-8.

18. Sinclair H, Bond C, Lennox A, Silcock J, Winfield A, Donnan P. Training pharmacists and pharmacy assistants in the stage-ofchange model of smoking cessation: a randomised controlled trial in Scotland. Tobacco Control 1998;7:253-61.

19. Machado M, Bajcar J, Guzzo GC, Einarson TR. The sensitivity of patient outcomes to pharmacist interventions. Part II: Systematic review and meta-analysis in hypertension management. Ann Pharmacother 2007;41:1770-81.

20. Morgado MP, Morgado SR, Mendes LC, Pereira LJ, Castelobranco $\mathrm{M}$. Pharmacist interventions to enhance blood pressure control and adherence to antihypertensive therapy: review and meta-analysis. Am J Health Syst Pharm 2011;68:241-53.

21. Santschi V, Chiolero A, Burnand B, Colosimo AL, Paradis G. Impact of pharmacist care in the management of cardiovascular disease risk factors a systematic review and meta-analysis of randomised trials. Arch Intern Med 2011;171:1441-53.

22. Santschi V, Chiolero A, Colosimo AL, Platt RW, Taffe P, Burnier M. Improving blood pressure control through pharmacist interventions: a meta-analysis of randomised controlled trials. J Am Heart Assoc 2014;3:e000718.

23. Cheema E, Sutcliffe P, Singer D. The impact of interventions by pharmacists in community pharmacies on control of hypertension: a systematic review and meta-analysis of 
randomised controlled trials. $\mathrm{Br} \mathrm{J}$ Clin Pharmacol 2014;78:1238-47.

24. Mohr DC, Ho J, Duffecy J, Reifler D, Sokol L, Burns MN, et al. Effect of telephone-administered vs face-to-face cognitive behavioural therapy on adherence to therapy and depression outcomes among primary care patients: a randomized trial. JAMA 2012;307:2278-85.

25. Wells K, Thornley T, Boyd M, Boardman H. Views and experiences of community pharmacists and superintendent pharmacists regarding the New Medicine Service in England prior to implementation. Res Soc Adm Pharm 2014;10:58-71.

26. Schulz M, Verheyen F, Muhlig S, Muller J, Muhlbauer K, KnopSchneickert E. Pharmaceutical care services for asthma patients: a controlled intervention study. J Clin Pharmacol 2001;41:668-76.

27. Barbanel D, Eldridge S, Griffiths C. Can a self-management programme delivered by a community pharmacist improve asthma control? A randomised trial. Thorax 2003;58:851-54.
28. Narhi U, Airaksinen M, Enlund H. Do asthma patients receive sufficient information to monitor their disease-a nationwide survey in Finland? Pharm World Sci 2001;23:242-5.

29. Herborg H, Soendergaard B, Jorgensen T, Fonnesbaek L, Hepler $\mathrm{C}$, Holst $\mathrm{H}$, et al. Improving drug therapy for patients with asthma-part 2. J Am Pharm Assoc 2001;41:551-9.

30. Cordina M, McElnay J, Hughes C. Assessment of a community pharmacy-based program for patients with asthma. Pharmacotherapy 2001;21:1196-203.

31. PGEU policy statement on adherence to medicines. Targeting adherence: Improving Patient Outcomes in Europe through Community Pharmacists' Intervention; 2008. Available from: http://www.pgeu.eu/policy/5-adherence.html. [Last accessed on 24 Aug 2016].

\section{How to cite this article}

- Ejaz Cheema, Paul Sutcliffe, Donald RJ Singer. Community pharmacist-led new medicine service for patients with a long term medical condition: a cross-sectional study. Int J Pharm Pharm Sci 2017;9(6):129-133. 